\title{
Beyond Enjoyment: A Cognitive-Emotional Perspective of Gamification
}

\author{
Jeffrey K. Mullins \\ University of Arkansas \\ jmullins@walton.uark.edu
}

\author{
Rajiv Sabherwal \\ University of Arkansas \\ rsabherwal@walton.uark.edu
}

\begin{abstract}
The success of gamified systems depends on their ability to engage players by eliciting both positive and negative emotions, but little guidance exists on creating emotional experiences through gamified design. This paper reviews work in psychology and neuroscience to highlight the interactive processes of cognition and emotion, and describes their relevance to gamification. Drawing on a model of the cognitive structure of emotions, and the mechanics-dynamics-emotions (MDE) framework for gamification, this paper advances a cognitive-emotional perspective of gamification and provides general propositions and directions for future research.
\end{abstract}

\section{Introduction}

"The importance of emotion to the variety of human experience is evident in that what we notice and remember is not the mundane but events that evoke feelings of joy, sorrow, pleasure, and pain. Emotion provides the principal currency in human relationships as well as the motivational force for what is best and worst in human behavior. Emotion exerts a powerful influence on reason and, in ways neither understood nor systematically researched, contributes to the fixation of belief" [17, p. 1991].

We read books that we can't put down, watch movies from which we can't look away, and engage in games that we can't stop playing. We experience a rollercoaster of emotions through these media: interest in an unfolding story, fear in dire situations, anger at antagonists, sadness in a time of loss, surprise from unexpected twists, disgust at disturbing imagery, and joy in eventual triumph. Emotions are central to the experience of engagement in literature [51], movies [67] and, more recently, games [45].

The most engaging games, like great works of fiction, evoke emotions in the player that vary in their nature, valence, and intensity. However, despite practitioners' recognition of the importance of emotions in games [31], scholars have only recently begun to study the complex interplay of positive and negative emotions in traditional game design [7] [45]. "Video games lead the way as interactive products that create emotion. More emotional than software and more interactive than films, games manipulate player affect to create poignant experiences" [38, p. 156]. This need for emotional depth also applies to the interrelated notions of a serious game [41], which is a full-fledged game designed for non-entertainment purposes [70], and gamification, which is defined as "the incorporation of game design elements into a target system while retaining the target system's instrumental functions" [40, p. 4]. Gamification seeks to enhance software and services through a "gameful" experience [28], in which some combination of conditions results in the subject perceiving that she or he is playing a game, whether or not the activity is normally associated with games [44].

The goals of gamification vary with the task(s) being gamified, and include increasing attention and engagement [14], stimulating innovation [9], improving decisions [24], promoting learning [32], and changing behavior [64]. We propose that emotion in the gamified experience is key to each such goal.

Prior work on gamification has focused on both psychological and behavioral outcomes, but the studied psychological outcomes have predominantly been motivational processes that are cognitive in nature [25]. Cognitive processes may include learning, attention, memory, and problem solving, among others [4]. Emotional processes, when studied in gamification, focus primarily on positive affect (e.g., enjoyment or fun) in a general sense. In both practitioner and academic literature on gamification, the general assumption is that positive affect is "good" and negative affect is "bad" (e.g., [44] [46]). While it is important to ensure that most gamified experiences are generally enjoyable, there is a need to understand how more specific emotions, both positive and negative, may help achieve the goals of gamification.

Advances in cognitive neuroscience have led to the view that "emotion and cognition are only minimally decomposable," and that behaviors are determined by complex and blurred interactions along multiple affective and cognitive dimensions [55, p. 155]. So, to complement the strong focus on cognitive processes in 
gamification research, a deeper understanding of relevant emotional processes is needed. Consistent with the premise that an effective gamified experience should evoke specific positive and negative emotions, which interact with cognitions to influence behavior, this paper seeks to offer a new theoretical perspective of gamification based on the interplay between cognition and emotion. To address this goal, we discuss the foundations of cognition and emotion in psychology and neuroscience, develop a cognitive-emotional perspective of gamification by examining the relationships among emotion, cognition, and gamification, and offer guidance for future research.

\section{Cognition and emotion}

Cognition refers to the mental activities pertaining to acquisition and application of knowledge, including processes such as attention, learning, language processing, problem solving, and memory. For decades, research on cognition has been dominated by a generally agreed-upon information processing view [35] [65] [66]. It is rooted in the work of Newell and Simon [49], likening the human brain to a computer that is capable of rapid serial processing of stimuli to achieve the goals of cognition.

Research on emotion has achieved less consensus regarding core tenets such as sources, frameworks, and even basic definitions of emotion. Here, we adopt the view that emotions are internal mental states of varying intensity representing evaluative reactions to environmental stimuli [52]. Various theories, illustrated in Table 1, have emerged to explain and categorize the complex landscape of emotions. While based on distinct premises, all recognize that emotions are adaptive, and as such are not inherently desirable or undesirable. Further, all account for common dimensions such as intensity, valence, and subjective feeling. The existence of a relationship between cognition and emotion is universally acknowledged, but the primacy of one over the other has been a topic of fundamental disputes in psychology [36] [39] [71].

Table 1. Emotion Theories

\begin{tabular}{|l|l|}
\hline Emotion Theory & Premise \\
\hline $\begin{array}{l}\text { Differential } \\
\text { Emotions Theory } \\
{[1][29]}\end{array}$ & $\begin{array}{l}\text { Innate (non-cognitive) emotions } \\
\text { develop early; learned (social- } \\
\text { cognitive) emotions develop later }\end{array}$ \\
\hline $\begin{array}{l}\text { Cognitive } \\
\text { Emotion Theory } \\
{[19][37]}\end{array}$ & $\begin{array}{l}\text { All emotions result from cognitive } \\
\text { appraisal, whether automatic or } \\
\text { volitional }\end{array}$ \\
\hline $\begin{array}{l}\text { Appraisal Theory } \\
{[60][63]}\end{array}$ & $\begin{array}{l}\text { Emotions result from unconscious } \\
\text { strategies for coping with particular } \\
\text { types of situations }\end{array}$ \\
\hline
\end{tabular}

A useful classification of emotions, advanced by Ortony, Clore, and Collins (OCC) [52], acknowledges the essential role of cognition in the structure of emotions. This model suggests that emotions are the result of a valenced reaction (positive or negative) to the consequences of events, actions of agents, or aspects of objects. Different emotions are triggered via each of these sources, and may be further subdivided on the bases of more granular conditions. For example, in considering conditions of other vs. self and desirable vs. undesirable events, the consequence of an event that is undesirable for some other may result in gloating (positive valence) or pity (negative valence). To resolve ambiguities in the original OCC model, a revised version has been developed [68]. We will return to this model later as a promising avenue for design guidance in gamification research and practice.

Research in neuroscience has begun to integrate emotion and cognition as inseparable influences in the neural processes that lead to behavior [17] [55] [56], and studied several processes at the intersection of cognition and emotion. Among the most basic of these processes is the relationship between emotion and attention via the amygdala, a brain region that has been primarily associated with emotion but is receiving increasing attention as a critical hub that regulates flow and integration of information between brain regions in cognitive-emotional interactions [54]. The amygdala is strongly linked to fear processing, and is proposed to modulate sensory processing via evolutionary mechanisms of self-preservation by focusing attention (a cognitive process) on potential threats [56]. The amygdala is also proposed to support processes of encoding, consolidation, and subjective recollection of memories linked to emotional stimuli [17] [56]. Other processes that are proposed to involve a complex interplay of cognition and emotion include emotional learning, processing of social stimuli, changing emotional responses, and decision making [55] [56]. We suggest that such processes are commonly experienced in games and gamification, and that theory and practice in gamification can be informed through greater attention to how these processes operate.

\section{Gamification}

Gamification is an emerging area of research in business and information systems [12] [40], finding outlets in highly-regarded journals (e.g., [12] [62]). Gamification of IS refers to the integration of game design elements into an existing system such that the system's instrumental functions are retained [40]. Elements of game design have been classified in a number of ways (e.g., [16] [64]), and are generally 
defined as the building blocks of which games are typically comprised. Examples of these elements include points, leaderboards, levels, badges, and challenges [16]. Game design elements may, individually or in combination, elicit specific emotions in the user, and these emotions can be harnessed to promote desired outcomes of the gamified experience. To date, however, little research has focused on the specific emotional outcomes of gamified experiences beyond the general premise that enjoyment and satisfaction are desirable, while distress and dissatisfaction are undesirable.

Gamification has generally led to positive outcomes [25], but some findings are mixed (e.g., [15]) or even show a negative influence of gamification (e.g., [27]). Failed efforts to gamify, estimated to be as high as 80 percent [20], are often attributed to poor game design [10]. It is through elements of game design that gameful experiences manifest, and these elements should interact to evoke a sense of emotional engagement in the player. Failure to gamify is a failure to create gameful experiences through careful and deliberate design.

A recent framework of gamification design, illustrated in Figure 1, incorporates mechanics, dynamics, and emotions (MDE) [59]. Mechanics comprise the "designed" aspects of the gamified system, including goals, rules, contexts, boundaries, and types of interactions that are possible. Three types of mechanics are proposed in the MDE framework. Setup mechanics refer to the context of the experience (e.g., single- or multi-player, available objects in the game). Rule mechanics refer to the goals, allowable actions, and constraints (e.g., time limits, achievement criteria). Progression mechanics refer to the rewards and reinforcements that are used to influence player behavior (e.g., points, badges, and leaderboards).

Dynamics relate to the actions of the player, and are not under the direct control of designers. Players may approach a game with different strategies, and may react to game mechanics in different ways. Dynamics are difficult to predict, and it is through dynamics that unintended consequences of gamification can arise.

The MDE framework takes an important initial step toward highlighting the importance of emotional experiences in motivating human behavior. Consistent with prior work on gamification, the MDE framework proposes that enjoyment is the single most important player engagement goal, and that enjoyment may come from a variety of positive emotions such as excitement, surprise, and triumph over adversity. Extending this premise, MDE acknowledges the importance of mixed emotions such as disappointment or sadness resulting from failures within the game. While MDE suggests that designers should focus first on controlling the experience through mechanics, then on dynamics, and lastly on players' emotions, it inversely suggests that, for players, emotions are "more important than the rules that make them possible" [59, p. 416].

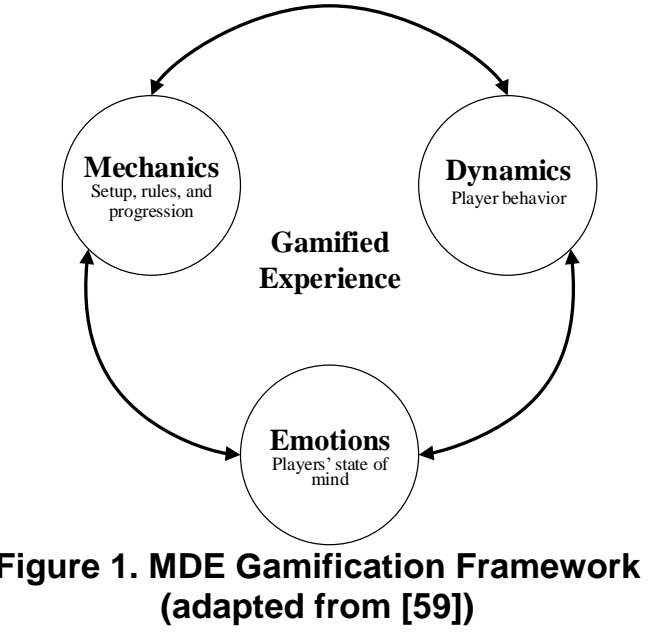

Consistent with the view that both emotions and cognitions are of paramount importance in a gamified experience, in the following section we integrate insights from psychology and cognitive neuroscience to suggest a new theoretical perspective for the study of gamification, draw on the MDE framework and OCC model to guide the design of gameful cognitive and emotional experiences, and offer directions for future research using a cognitive-emotional lens.

\section{A cognitive-emotional perspective of gamification}

Current approaches to the study of gamification adopt psychological perspectives based on traits, behavioral learning, cognition, self-determination, interest, or emotion [61]. We suggest that a perspective integrating cognitions and emotions offers greater opportunities for theoretical inquiry and practical application. Drawing on the relevant neuroscience literature, we introduce such a perspective with four areas of inquiry that may be fruitful in the design and assessment of gamified systems. We then adapt a theory of the cognitive structure of emotions [52] to the domain of gamification and suggest mechanics through which specific emotions may be engendered by means of the thoughtful application of game design elements.

\subsection{Emotion and learning}

Classical stimulus-response conditioning [53] has been associated with various types of learning for much of the last century [5] [23]. A stimulus response to induced fear leads to physiological reactions that are 
processed through the amygdala - this has been shown to hold for fears acquired directly, through language, or observed vicariously [56]. Such associative learning through emotional stimuli has been linked to the cognitive process of learning [55]. Some evidence also indicates in both human and non-human subjects that positive stimuli, such as rewards, may facilitate emotion-driven learning [56]. Also, the consolidation process through which memories become stable over time is enhanced by emotional arousal, because such memorable events are more likely to be important to survival from an evolutionary perspective [56]. In the context of video games, the view of games as "controlled training regimens" is supported by growing evidence that performance improvements resulting from video games are "paralleled by enduring and functional neurological remodeling” [6, p. 763].

Around one quarter to one third of studies on gamification are conducted in the domain of education [25] [64]. Thus, the importance of learning as an outcome of gamification is underscored in our presentation of a cognitive-emotional perspective. To the extent that the design of gameful experiences can promote acquisition of knowledge by eliciting specific emotions in the player, researchers and practitioners should investigate the contextual and emotional factors under which learning is most effective. Salient contextual factors may include attributes of the individual (e.g., self-efficacy, achievement goal orientation), the task (e.g., complexity, predictability), or the technology (e.g., mobility, sensory feedback capability) [40]. Emotions that promote effective learning in one context may not be effective (or may even be detrimental) in another context.

Consider the example of an individual's achievement goal orientation. Individuals oriented toward performance goals are motivated by recognition of positive performance, while those oriented toward mastery goals are motivated by the opportunity to improve their abilities [57]. Providing poor performance feedback to an individual with a strong performance orientation may have a detrimental effect compared to providing the same performance feedback to an individual with a strong mastery orientation. While both individuals may experience an ostensibly "negative" emotion, a sense of disappointment paired with high mastery orientation may lead to the desired persistence in learning, while the same sense paired with a high performance orientation may lead to undesired frustration with the gamified experience.

\subsection{Emotion and memory}

As noted earlier, the amygdala is proposed to support the encoding, consolidation, and recollection of memories that are linked to emotional stimuli [56]. As part of the encoding process, the amygdala modulates the neural signal by imbuing it with additional import and information related to the emotional experience, facilitating later episodic recall of emotional material [55]. Memory and learning are closely related, with learning typically occurring as a result of effort over a period of time, and a memory representing a mentally stored representation of a specific occurrence at a point in time [33].

Gamification has been suggested to influence both working memory [50] and episodic memory [32]. Episodic memories are typically associated with strong associations with a particular time or place, so the potential for creating such memories through immersive games is high. To the extent that elements of game design can provide an immersive and memorable environment, and can trigger emotions that can facilitate recall of episodic memories, gamification may be able to harness this cognitive-emotional process to help achieve memory and learning goals [32]. For example, rewards have been studied as antecedents of episodic memory, with reward value and reward uncertainty proposed as factors in a reward signal [42]. In that study, the value of the reward was found to play a major role in modulating episodic memory, but the uncertainty was not. If a goal of a particular gamified design involves the need for a player to clearly remember a piece of information, the association of a valued reward with an immersive experience may be an effective mechanism for supporting this goal. As with learning and emotion, effects such as these may also be contingent on the context of the person, task, and technology.

\subsection{Emotion and attention}

Attentional resources are highly valuable and seem to be increasingly scarce. In situations involving limited attentional resources, stimuli that evoke emotional responses are more likely to capture attentional focus [17] [56]. Automatic processing of emotional stimuli is generally acknowledged [71], particularly in response to fear or threat conditions. More specifically, activity in the amygdala is highly correlated with activity in the visual cortex, such that "increasing the affective significance of a stimulus in a manner that is believed to be strongly amygdala-dependent has effects that are similar to those of increased attention" [54, p. 149]. Emotion may also be "preattentive," such that subliminal emotional stimuli still result in expected physiological responses [17]. Additionally, emotion is suggested to prevent "inattentional blindness," which refers to the tendency to miss a second stimulus after detecting an initial visual stimulus [17]. 
From the perspective of games and gamification, first-person shooter games have been shown to lead to faster and more accurate attention allocation [22]. Meaningful engagement, in which the dual outcomes of fostering engagement and enhancing instrumental task outcomes are met, has been proposed as a primary objective of gamification [40]. Focused attention is a key element in achieving a state of cognitive absorption or "flow" [13] [3]. One framework in the literature on games suggests three levels of flow: engagement (e.g., attraction and investment), engrossment (e.g., narrowed focus and increased emotional involvement), and total immersion (e.g., experience of presence and empathy) [8]. During a state of total immersion in a game, greater levels of anxiety and other negative emotions are experienced, and these negative emotions are suggested to increase levels of engagement [30]. To the extent that elements of game design can evoke emotions that facilitate a deeper state of flow, gamification may be able to harness this cognitive-emotional process to provide engaging and enjoyable experiences for players. Caution should be exercised when promoting deeper levels of engagement, as at least one study has found that greater levels of emotional and subjective attachment in game-based science learning can lead to less reliable learning outcomes [11].

For example, challenge (a design element representing a difficult in-game task) has been shown to impact engagement, immersion, and perceived learning in a game-based learning context, and engagement (but not immersion) was also found to affect perceived learning [26]. A challenge presents players with a difficult task, and may evoke negative emotions such as frustration and anxiety during attempts to meet the challenge. It is precisely this type of emotional involvement, within a certain range of intensity, which promotes the experience of immersion [30]. While an immersive experience is known to lead to greater levels of enjoyment, the level of emotional arousal should be managed carefully to promote "just enough" emotion to maximize meaningful engagement, such that neither user engagement nor instrumental outcomes are compromised.

\subsection{Emotion and decision making}

Cognitive and emotional processes are also integrated in executive control [54] and decision making [17]. Judgment is improved as a result of enhanced bodily states stemming from emotional arousal, such that the recollection of prior feeling states can bias decision making through anticipation of reward or punishment [17]. The role of the amygdala is central in this process, interacting with the pre-frontal cortex to compute expected rewards resulting from decision options [55]. Emotion also plays an important role in processing social stimuli, such as the recognition of emotions in the faces and actions of others - an important factor in decision making [56].

The range of negative emotions triggered by games includes frustration, anger, anxiety, and sadness, and the "pretend context of video games may be real enough to make the accomplishment of goals matter but also safe enough to practice controlling, or modulating, negative emotions in the services of those goals" [22, p. 72]. This balance of an imaginary context and real emotions leads to more adaptive regulation strategies such as problem solving and reappraisal as players learn to deal with negative emotions in adaptive ways [22]. Strategy games, typically implemented as simulations of some complex process such as civilization building or warfare, have been shown to create cognitive scaffolding to support decision-making [47] and improve self-reported problem solving skills [2].

Consider the design element of limited resources, in which the player must prioritize goals and make decisions under constrained conditions. Such a constraint may lead to fear of making suboptimal decisions, and relief when the decision works out (or disappointment when it doesn't). In the perceived context of a game, however, the player will have the opportunity to work through the variety of emotions and cognitions in a "safe" place within the gamified system.

\section{Designing for emotion}

To offer initial guidance on the process of designing gamification mechanics to elicit specific emotions, we draw on the cognitive structure of emotions [52] [68]. This theory relates to the underlying structure of emotions and causal chains through which they emerge. While certain linguistic tokens (emotion words) are used to represent types of emotions in the proposed structure, these tokens are only one component of the theory. The theory begins with the premise that there are three major aspects of the world that are subjected to changes from the perspective of a given individual: events, agents, and objects. For any change to one of these aspects, that individual may experience a valenced reaction of a certain type and of variable intensity depending on a number of factors in the environment. This valenced reaction may be related to the consequences of events, the actions of agents, or the aspects of objects.

At a high level, a reaction to a consequence of some event (e.g., earning a badge) may be a coarse-grained sense of pleasure (positive valence) or displeasure (negative valence). If the focus is on a referent other (e.g., another player earning the badge), the experienced emotion becomes more specific, such as resentment or 
being happy for the other individual. Figure 2 presents the revised OCC model [68]. Terms that are contained within boxes in the figure represent emotions resulting from the conditions indicated above each, with emotions listed lower in the figure being more specific to conditions indicated earlier in the chain. Each emotion type is represented by example terms for a positive (top) and negative (bottom) valenced response. The dotted lines (added) represent the scope of propositions (P1$\mathrm{P} 3$ ) which will be developed in the following sections.

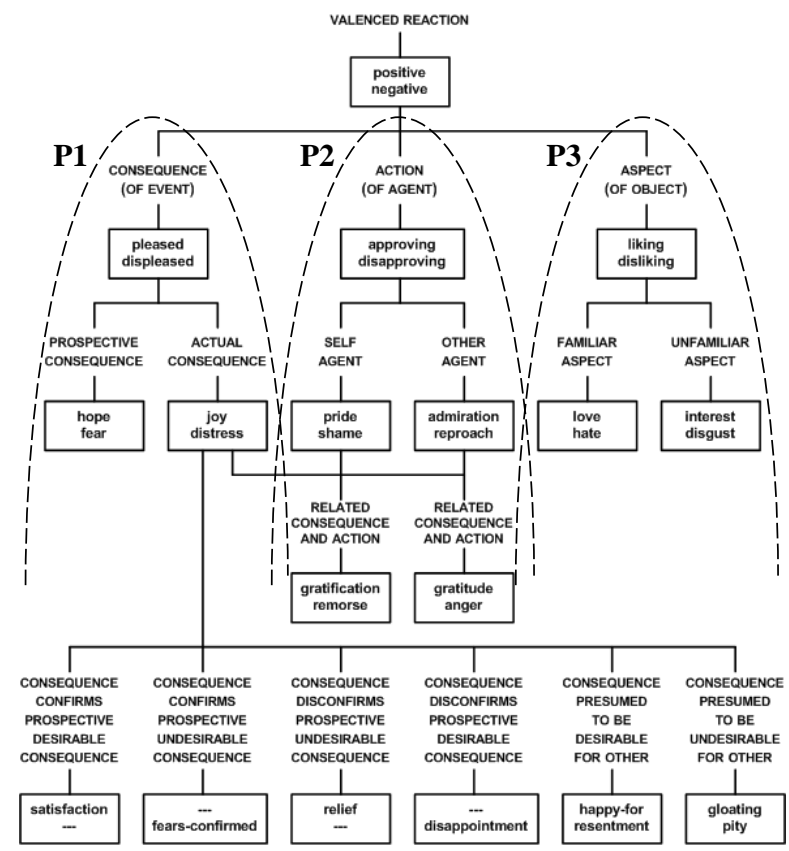

Figure 2. Revised OCC Model [68]

Given that a key design goal for this model is to provide a computationally tractable system that can be used to support artificial intelligence applications [52], we suggest that this model provides a useful starting point for determining how to evoke particular emotions through game design. For example, if the desired cognitive process can be augmented by a sense of fear in the player, based on this structure it would be advisable to create some event that portends a negative prospective consequence for the player.

The MDE framework proposes three categories of game mechanics that are present in all games and gamified experiences (setup, rule, and progression) [59]. Dynamics must also be considered, but are outside of the direct control of designers. Emotions, proposed as the final consideration for designers but the most important aspect for the player, are addressed in greater detail in the sections below. While some consideration of mechanics is necessary in the early stages of any gamified design, we suggest that the desired emotional outcomes be considered first, and that those considerations should play a role in the mechanics and target dynamics of the gamified experience.

\subsection{Consequences of events}

In order to evoke a sense of pleasure or displeasure in a player, there must be some mechanic to generate an event with a relevant consequence. This mechanic may be related to the setup, for example via random generation of the player's in-game character's attributes. If those attributes are desirable but of no actual consequence in future interactions (e.g., a character's virtual appearance), then emotions at a deeper level than general pleasure or displeasure are unlikely to be elicited. If those attributes offer prospective future consequences (e.g., a number of virtual "lives"), such a mechanic may generate a sense of hope or fear. When those consequences are actualized (e.g., gaining or losing a life), this may shift to a sense of joy or distress. Thus, if the goal is to evoke a sense of joy, a gamification mechanic should instantiate an event with a desirable consequence in the gamified environment.

Rule mechanics can also evoke emotions through consequences of events. In mobile applications, rule mechanics may involve consequences of geo-location or physiological monitoring, such as earning badges for checking in or accumulating points for physical activity. For example, the "activity rings" on the popular Apple Watch $^{\circledR}$ product are embedded with a set of rule mechanics for making progress toward daily fitness goals. As a wearer exercises, he or she may develop a sense of fear that the current exercise routine will not be enough to meet the daily objective. Thus, if the goal is to evoke a sense of fear, a gamification mechanic should create conditions with prospective negative consequences (which may be implicit or explicit).

Progression mechanics, representing the rewards and incentives tied to players' actions in a gamified experience, can evoke emotions through consequences of events as well. Rewards may include points, badges, social status, physical rewards, etc. Awarding a badge, for example, is only likely to lead to satisfaction if some prior hope felt by the player is confirmed by the actual consequence of earning the badge. If the player did not have prior sense of hope to earn the badge, it is more likely that the emotional outcome will be a more general sense of pleasure. Thus, to evoke a sense of satisfaction, it is necessary to first create awareness of the consequence and to ensure that the consequence is of relevance to the player.

Proposition 1: Gamification mechanics should be aligned with the desired emotional outcome, such that prospective and actual consequences of an event, and the confirmation of such consequences, are incorporated consistently with the revised OCC model. 


\subsection{Actions of agents}

Actions of agents represent a potentially difficult set of conditions through which to elicit emotions in a player. Because the dynamics of player choices and behaviors are unpredictable, the focus of gamified mechanics should be based on either 1) probabilistic player responses to setup, rule, and progression mechanics, or 2) more scripted and controlled behaviors of non-player characters (NPCs). In the case of the former, especially for multi-player environments, game mechanics should be designed to encourage or discourage certain types of actions in order to maximize the probability that desired emotions will be achieved. In the case of the latter (NPCs), the ability of designers to control the behavior of the "other agent" affords more opportunity to evoke specific emotions, but the intensity of experienced may be lower when the player knows that they are interacting with a machine.

Setup mechanics may establish how many agents are involved, the nature of those agents (real or NPC), and may assign initial conditions to those agents such as locations or roles. For example, consider a scenario in which the software development function in an organization is gamified, and that one of the "players" is assigned a role as a "spy." By conducting covert code reviews, the spy may experience a sense of pride in the role, while others may react to the actions of the spy (uncovering bugs in their code) with either gratitude or anger. Thus, to elicit a sense of gratitude, game design mechanics must be in place to support and encourage the interactions necessary to identify agents, observe their actions, and favorably evaluate the consequences of those actions.

Rule mechanics are instrumental in enabling and encouraging certain types of interactions among players, but the dynamics of the experience cannot guarantee that the desired emotional state will be achieved. Returning to the example of the software spy, some type of rule mechanic may be in place to initially protect the spy from detection, and another to eventually uncover the spy's identity through the actions of other agents or after a period of time. Actions of agents will be informed by rule mechanics and those actions may subsequently be perceived by others on the basis of conditions consistent with the revised OCC model.

Progression mechanics will relate indirectly to the actions of agents, as they typically provide a signal of progression as a consequence of an event, which may have occurred as a result of the action of an agent. Returning again to the example of the software spy, if the spy completes a mission to identify a certain number of bugs in a given time period, and the goal is achieved, this could result in a sense of gratification on the part of the spy, or a deeper sense of relief if the mission was accomplished without the undesirable consequence of being detected. These emotions are related to both the actual consequences of events and the related consequences of agent actions in the OCC model.

Proposition 2: Gamification mechanics should be aligned with the desired emotional outcome, such that the actions of human agents are appropriately enabled and constrained to encourage the desired emotional states through the ability to identify agents, observe their actions, and recognize the consequences of those actions consistently with the revised OCC model.

\subsection{Aspects of objects}

Aspects of objects provide opportunities to elicit a more limited, but also a critically important, set of emotions to achieve player engagement. Beyond general liking or disliking, the primary condition in differentiating emotions resulting from the aspects of objects is the aspect's familiarity, with familiar aspects resulting in love or hate, and unfamiliar aspects resulting in interest or disgust.

Setup mechanics are proposed to play the strongest role in eliciting this set of emotions, as it is the setup mechanics which dictate what objects (and aspects of objects) will be available in the gamified experience. The setup mechanic of progressively advanced levels, for example, draws on unfamiliar aspects of the game to spark and maintain interest in the player. Setup mechanics can also affect the intensity of experienced emotions. For example, a sensory environment with high-resolution graphics, realistic audio, and haptic feedback enables a rich and immersive gameful experience. The realism of such an environment is likely to affect the intensity of emotions experienced.

Rule mechanics may support emotional experiences in gamification to the extent that they alter and highlight aspects of objects, but are also constrained by the setup mechanics shaping the availability and composition of objects in the gamified environment. A rule mechanic may, for example, unlock a new level on the basis of some achievement in the game. While the new level (a setup mechanic) may serve the purpose of evoking interest, and the achievement (a progression mechanic) may lead to a sense of joy, the actions of the player in accordance with rule mechanics in achieving the goal are integral in generating emotions such as pride, gratification, and relief.

Progression mechanics may directly or indirectly impact emotions that result from evaluating the aspects of objects in the gamified environment. For example, challenges (a progression mechanic) issued by a mobile fitness app may involve familiar aspects that are liked or disliked by the player. A challenge to complete a five kilometer run may be appealing to one player, while a 
challenge to complete fifty push-ups in a day may be unappealing to the same player. The very existence of the challenge and its possible aspects are reflected in setup mechanics, while the instantiation of the challenge is controlled by progression mechanics.

Proposition 3: Gamification mechanics should be aligned with the desired emotional outcomes, such that the player's familiarity with objects and their aspects is incorporated consistently with the revised OCC model.

Taken together, we propose a cognitive-emotional theoretical perspective of gamification. Informed by advances in cognitive neuroscience on the integrated nature of cognition and emotion in the determination of behavior, the emotions that support particular types of cognition are highlighted. To link these emotions to gamified design, we integrate the revised OCC model of emotions with the MDE gamification framework and suggest propositions for aligning the desired emotional outcomes with relevant setup, rule, and progression mechanics in the design of gamified systems.

\section{Discussion}

This paper makes several contributions to the gamification literature. We have addressed calls for greater theorizing around gamification [40] [64] by advancing a cognitive-emotional theoretical perspective that can guide the design of gamified systems to elicit the desired emotions in support of cognitive goals. We draw specific attention to the importance of both positive and negative emotions in creating an engaging experience through gamification. In doing so, we focus on one theory of emotions (OCC) in a large body of diverse emotion literature, and believe additional insights could be gained from integrating alternate theories of emotion.

We integrate a model of the cognitive structure of emotions (OCC) with a framework for gamification (MDE) to offer an initial explication of how certain types of mechanics may be used to elicit specific types of emotions. This perspective provides a theoretical contribution by drawing on work in neuroscience to inform gamification research, by explaining how elements of game design can interact with both emotion and cognition to produce desired outcomes.

This paper also makes contributions to practice in the area of gamification. First, it provides guidance for designers of gamified systems. By integrating a "map" of the structure of emotions with the types of game mechanics that can be used to elicit various emotions, designers can draw on this perspective for insights to increase the likelihood of a successful gamified design. Also, this perspective may be valuable in identifying effective patterns of emotional arousal in existing games and gamified systems, leading to the formulation of templates for creating desired emotional experiences.

However, we need to exercise caution in moving forward to unpack the mysteries of emotion to influence human behavior through gamification. Emerging literature examines ethical issues in gamification (e.g., [34] [69]). Care must be taken to maintain awareness of potential ethical issues in gamification, and develop and adhere to standards of ethical behavior.

We hope that this work can motivate further inquiry in the area of emotions and gamification, and suggest opportunities for further research. One research implication relates to the empirical methods used to measure emotions. For example, measures of heart rate when playing computer games may increase in response to emotional arousal, but drop in response to greater attentional engagement, both of which are considered indicators of engagement in games [58]. Pairing physiological measures with self-report data, and triangulating between multiple physiological and neurological measures, can help to avoid associated measurement risks.

Recent work has begun to acknowledge the need for more complex emotional experiences in achieving deeper levels of engagement persuasion through engineered experiences. A call for "serious experience" in serious games provides one example of this need [41]. Similar calls have been made for the design of everyday objects [18] and persuasive health messages [48]. Future gamified experiences could become carefully engineered experiences designed to evoke specific emotions and cognitions at desired levels of intensity, in the appropriate sequence, and matching the targeted cognitions to achieve the desired outcome.

The investigation of contextual differences is especially important in the domain of emotions. Perhaps most important are differences among individuals, which may reflect task-relevant attributes (e.g., goal orientation in a learning task), general attributes (e.g., self-efficacy, trait anxiety), or attributes reflecting how individuals manage emotions (e.g., emotional intelligence [21] [43]).

\section{Conclusion}

Emotion represents significant uncharted territory in the area of gamification, given the importance of emotional engagement in the creation of gameful experiences. We offer a fresh theoretical lens on gamification which integrates literature in psychology and neuroscience to better understand the alignment of desired cognitions, emotions, and gamification mechanics. Gamified experiences, like games, should be enjoyable. However, the enjoyment of a gameful 
experience, like the enjoyment of literature or film, involves both positive and negative emotions. In order to support the instrumental functions of a system while also engaging the player in a gameful experience, it is necessary to simultaneously consider cognition and emotion in the design of gamified systems.

\section{References}

[1] Ackerman, B. P., Abe, J. A., and Izard, C. E. "Differential emotions theory and emotional development", What develops in emotional development, 1998, 85-106.

[2] Adachi, P. J., and Willoughby, T. "More than just fun and games: The longitudinal relationships between strategic video games, self-reported problem solving skills, and academic grades", Journal of Youth Adolescence, 42, 2013, 1041-1052. [3] Agarwal, R., and Karahanna, E. 'Time flies when you're having fun: Cognitive absorption and beliefs about information technology usage", MIS quarterly 24(4), 2000, 665-682.

[4] Anderson, J. R. Cognitive psychology and its implications, Henry Holt \& Co., New York, 1990.

[5] Anderson, J. R. Learning and memory, Wiley, New York, 2000.

[6] Bavelier, D., Green, C. S., Han, D. H., Renshaw, P. F., Merzenich, M. M., and Gentile, D. A. "Brains on video games", Nature reviews neuroscience, 12(12), 1990, 763-768. [7] Bopp, J. A., Mekler, E. D., and Opwis, K. "Negative emotion, positive experience? Emotionally moving moments in digital games", in CHI '16 Proceedings, 2016, 2996-3006.

[8] Brown, E., and Cairns, P. "A grounded investigation of game immersion", in $\mathrm{CHI}$ '04 extended abstracts on human factors in computing systems, 2004, 1297-1300.

[9] Burke, B. "Gartner says by 2015, more than 50 percent of organizations that manage innovation processes will gamify those processes", http://www.gartner.com/newsroom/id/1629214, 2011.

[10] Burke, B. Gamify: How gamification motivates people to do extraordinary things, Routledge, New York, 2014.

[11] Cheng, M. T., She, H. C., and Annetta, L. A. "Game immersion experience: Its hierarchical structure and impact on game-based science learning", Journal of computer assisted learning, 31(3), 2015, 232-253.

[12] Colbert, A., Yee, N., and George, G. "The digital workforce and the workplace of the future", Academy of management journal, 59(3), 2016, 731-739.

[13] Csikszentmihalyi, M. Beyond boredom and anxiety, Jossey-Bass, San Francisco, 1975.

[14] Dale, S. "Gamification: Making work fun, or making fun of work?" Business information review, 31(2), 2014, 82-90.

[15] de-Marcos, L., Domínguez, A., Saenz-de-Navarrete, J., and Pagés, C. "An empirical study comparing gamification and social networking on e-learning", Computers \& education, 75, 2014, 82-91.

[16] Deterding, S., Dixon, D., Khaled, R., and Nacke, L. "From game design elements to gamefulness: Defining gamification," in Proceedings of the 15th international academic MindTrek conference: Envisioning future media environments, 2011, 9-15.
[17] Dolan, R. J. "Emotion, cognition, and behavior", Science, 298(5596), 2002, 1191-1194.

[18] Fokkinga, S., and Desmet, P. "Darker shades of joy: The role of negative emotion in rich product experiences", Design issues, 28(4), 2012, 42-56.

[19] Frijda, N. H. "Impulsive action, and motivation", Biological psychology, 84, 2010, 570-579.

[20] Gartner. "Gartner says by 2014, 80 percent of current gamified applications will fail to meet business objectives primarily due to poor design (Press Release)", http://www.gartner.com/newsroom/id/2251015, 2012.

[21] Goleman, D. Emotional intelligence, Bantam, New York, 2006.

[22] Granic, I., Lobel, A., and Engels, R.C. "The benefits of playing video games", American psychologist, 69(1), 2014, 66-78.

[23] Guthrie, E. R. "Conditioning as a principle of learning”, Psychological review, 37(5), 1930, 412-428.

[24] Hamari, J., and Koivisto, J. "Social motivations to use gamification: An empirical study of gamifying exercise", in ECIS proceedings, 2013, 105.

[25] Hamari, J., Koivisto, J., and Sarsa, H. "Does gamification work? - A literature review of empirical studies on gamification", in Proceedings of the 47th Hawaii International Conference on System Sciences, 2014.

[26] Hamari, J., Shernoff, D. J., Rowe, E., Coller, B., AsbellClarke, J., and Edwards, T. "Challenging games help students learn: An empirical study on engagement, flow and immersion in game-based learning", Computers in human behavior, 54, 2016, 170-179.

[27] Hanus, M. D., and Fox, J. "Assessing the effects of gamification in the classroom: A longitudinal study on intrinsic motivation, social comparison, satisfaction, effort, and academic performance", Computers \& education, 80, 2015, 152-161.

[28] Huotari, K., and Hamari, J. "Defining gamification: a service marketing perspective", in Proceeding of the 16th International Academic MindTrek Conference, 2012, 17-22.

[29] Izard C. E. "Basic emotions, relations among emotions, and emotion-cognition relations", Psychological review, 99, 1992, 561-565.

[30] Jennett, C., Cox, A. L., Cairns, P., Dhoparee, S., Epps, A., Tijs, T., and Walton, A. "Measuring and defining the experience of immersion in games", International journal of human-computer studies, 66(9), 2008, 641-661.

[31] Kane, B. "34 ways to put emotions into games", presentation at Game Developers Conference 2003 from http://www.gamasutra.com/view/feature/131293/34_ways_to _put_emotions_into_games.php, 2003.

[32] Kapp, K. M. The gamification of learning and instruction: Game-based methods and strategies for training and education, John Wiley \& Sons, New Jersey, 2012.

[33] Kazdin, A.E. (Ed.) Encyclopedia of psychology (Vols. 18), American Psychological Association, Washington, DC, 2000 .

[34] Kim, T. W. "Gamification of labor and the charge of exploitation”, Journal of business ethics, 2016, 1-13.

[35] Lachman, R., Lachman, J. L., and Butterfield, E. C. Cognitive psychology and information processing: An introduction, Psychology Press, New York, 2003. 
[36] Lazarus, R.S. "Thoughts on the relations between emotion and cognition", American psychologist, 37(9), 1982, 1019-1024.

[37] Lazarus, R. S., and Folkman. S. Stress, appraisal and coping, Springer Publishing, New York, 1984.

[38] Lazzaro, N. "Why we play: Affect and the fun of games", in Sears, A., and Jacko, J. A. (eds.), Human-computer interaction: Designing for diverse users and domains, 2009, $155-176$.

[39] Leventhal, H., and Scherer, K. "The relationship of emotion to cognition: A functional approach to a semantic controversy", Cognition and emotion, 1(1), 1987, 3-28.

[40] Liu, D., Santhanam, R., and Webster, J. "Towards meaningful engagement: A framework for design and research of gamified information systems", MIS quarterly, forthcoming.

[41] Marsh, T., and Costello, B. "Experience in serious games: Between positive and serious experience", in Serious Games Development and Applications (SGDA 2012) conference proceedings, 2012, 255-267.

[42] Mason, A., Farrell, S., Howard-Jones, P., and Ludwig, C. J. "The role of reward and reward uncertainty in episodic memory", Journal of memory and language, 96, 2017, 62-77. [43] Mayer, J. D., Salovey, P., and Caruso, D. R. "Emotional intelligence: New ability or eclectic traits?",American psychologist, 63(6), 2008, 503-517.

[44] McGonigal, J. Reality is broken: Why games make us better and how they can change the world, Penguin, London, 2011.

[45] Mekler, E. D., Rank, S., Steinemann, S. T., Birk, M. V., and Iacovides, I. "Designing for emotional complexity in games: The interplay of positive and negative affect", in Proc. of the 2016 Annual Symposium on Computer-Human Interaction in Play Extended Abstracts, 2016, 367-371.

[46] Mollick, E.R. and Rothbard, N. "Mandatory fun: Consent, gamification and the impact of games at work", Wharton School research paper series, https://ssrn.com/abstract=2277103, 2014.

[47] Morris, B., Croker, S., Zimmerman, C., Gill, D., and Romig, C. "Gaming science: The "gamification" of scientific thinking", Frontiers in psychology, 4, 2013.

[48] Nabi, R. L. "Emotional flow in persuasive health messages", Health communication, 30(2), 2015, 114-124.

[49] Newell, A., and Simon, H. A. Human problem solving, Prentice-Hall, New Jersey, 1972.

[50] Ninaus, M., Pereira, G., Stefitz, R., Prada, R., Paiva, A., Neuper, C., and Wood, G. "Game elements improve performance in a working memory training task", International journal of serious games, 2(1), 2015, 3-16.

[51] Oatley, K. "A taxonomy of the emotions of literary response and a theory of identification in fictional narrative", Poetics, 23(1-2), 1995, 53-74.

[52] Ortony, A., Clore, G. L., and Collins, A. The cognitive structure of emotions, Cambridge University Press, 1990.

[53] Pavlov, I. P. Conditioned reflexes: An investigation of the physiological activity of the cerebral cortex, Oxford University Press, London, 1927.
[54] Pessoa, L. "On the relationship between emotion and cognition", Nature reviews neuroscience, 9(2), 2008, 148158.

[55] Pessoa, L. The cognitive-emotional brain: From interactions to integration, MIT press, Cambridge, 2013.

[56] Phelps, E. A. "Emotion and cognition: Insights from studies of the human amygdala", Annual Review of Psychology, 57, 2006, 27-53.

[57] Pintrich, P. R. "The role of goal orientation in selfregulated learning", in Boekaerts, M., Pintrich, P. R., and Zeidner, M. (eds.), Handbook of Self-Regulation, Academic Press, San Diego, 2000, 451-502.

[58] Ravaja, N., Saari, T., Turpeinen, M., Laarni, J., Salminen, M., and Kivikangas, M. "Spatial presence and emotions during video game playing: Does it matter with whom you play?" Presence: Teleoperators and Virtual Environments, 15(4), 2006, 381-392.

[59] Robson, K., Plangger, K., Kietzmann, J. H., McCarthy, I., and Pitt, L. "Is it all a game? Understanding the principles of gamification", Business horizons, 58(4), 2015, 411-420.

[60] Roseman, I. J. "Appraisal in the emotion system: Coherence in strategies for coping," Emotion Review, 5(2), 2013, 141-149.

[61] Sailer, M., Hense, J., Mandl, H., and Klevers, M. "Psychological perspectives on motivation through gamification", in $I x D \& A, 19,2013,28-37$.

[62] Santhanam, R., Liu, D., and Shen, W. C. M. "Gamification of technology-mediated training: Not all competitions are the same", Information systems research, 27(2), 2016, 453-465.

[63] Scherer, K. R. “Appraisal theory," in Dalgleish, T., and Power, D. (eds.), Handbook of Cognition and Emotion, John Wiley \& Sons, West Sussex, England, 1999, 637-663.

[64] Seaborn, K., and Fels, D. I. "Gamification in theory and action: A survey", International journal of human-computer studies, 74, 2015, 14-31.

[65] Shiffrin, R. M., and Schneider, W. "Controlled and automatic human information processing: II. Perceptual learning, automatic attending and a general theory", Psychological review, 84(2), 1977, 127-190.

[66] Simon, H. A. "Information processing models of cognition", Annual review of psychology, 30(1), 1979, 363396.

[67] Smith, G. M. Film structure and the emotion system, Cambridge University Press, 2003.

[68] Steunebrink, B. R., Dastani, M., and Meyer, J. J. C. "The OCC model revisited", in Proc. of the 4th Workshop on Emotion and Computing, 2009.

[69] Thorpe, A. S., and Roper, S. "The ethics of gamification in a marketing context", Journal of business ethics, 2017, 113.

[70] Walz, S. P., and Deterding, S. The gameful world: Approaches, issues, applications, MIT Press, Cambridge, 2015.

[71] Zajonc, R. B. "Feeling and thinking: Preferences need no inferences", American psychologist, 35(2), 1980, 151-175. 